\title{
Como se escreve, no Brasil, a História da Psicologia no contexto hospitalar?
}

\section{How is it written, in Brazil, the History of Psychology in the hospital context?}

Denis Barros de Carvalho*

Universidade Federal do Piauí - UFPI, Teresina, Piauí, Brasil

\section{Leylanne Martins Ribeiro de Souza**}

Universidade Estadual do Piauí - UESPI, Teresina, Piauí, Brasil

\section{Lourena Silva Rosa**}

Universidade Estadual do Piauí - UESPI, Teresina, Piauí, Brasil

\author{
Máyra Laís de Carvalho Gomes** \\ Universidade Estadual do Piauí - UESPI, Teresina, Piauí, Brasil
}

\begin{abstract}
RESUMO
O objetivo deste ensaio é analisar a produção historiográfica brasileira sobre a inserção da psicologia no contexto hospitalar à luz dos conceitos de História Crítica de Karl Danziger. O texto divide-se em quatro partes: Inicialmente, uma revisão sobre os estudos acerca da Psicologia da Saúde no contexto hospitalar é apresentada; o núcleo central do trabalho é a segunda seção, na qual se apresenta uma análise das pesquisas sobre a História da Psicologia no contexto hospitalar; na próxima seção se busca repensar a pesquisa histórica sobre a Psicologia no contexto hospitalar a partir de uma História Crítica da Psicologia da Saúde.

Palavras-chave: História da Psicologia; Psicologia da saúde no contexto hospitalar; Trabalho do psicólogo.
\end{abstract}

\begin{abstract}
The objective of this essay is to analyze the Brazilian historiographical production on the insertion of Psychology in the hospital context to the light of the concepts of critical history of Karl Danziger. The text is divided in four parts: Initially, a revision on the studies concerning the Psychology of the Health in the hospital context is presented; the central nucleus of the work is the second section, in which if it presents an analysis of the research on the History of Psychology in the hospital context; in the next section if it searchs to rethink the historical research on Psychology in the hospital context from a Critical History of the Psychology of the Health.
\end{abstract}

Keywords: History of Psychology, Psychology of the health in the hospital context, The work of the psychologist. 


\section{I ntrodução}

A história da Psicologia, como área de pesquisa e campo de conhecimento, passou por grandes transformações nos últimos quarenta anos. O crescimento exponencial do número de publicações (artigos, livros didáticos, manuais, etc.) indica essa mudança quantitativa (HILGARD; LEARY; MCGUIRE, 1998).

A ênfase inicial na História da Psicologia como Ciência e em uma História puramente intelectual vem sendo substituída por uma investigação que pluralizou seus interesses, apresentando interessantes estudos sobre a profissionalização da Psicologia (GEUTER, 1992; CAPSHEW, 1999), o impacto social dos testes psicológicos (SOKAL, 1987; ZENDERLAND, 1998; DERKSEN, 2001; GIBBY; ZICKAR, 2008), relação da Psicologia com o racismo (RICHARDS, 1997; GUTHRIE, 1998), a relação da Psicologia com a sociedade (O'DONNELL, 1985; ASH; WOODWARD, 1989; CUSHMAN, 1995), a internacionalização da Psicologia (BROCK, 2006; PICKREN, 2009) e o contexto social da produção do conhecimento psicológico (DANZIGER, 1994), para citar alguns exemplos.

Essa mudança qualitativa na produção historiográfica sobre Psicologia decorre de um intenso questionamento do que se denominou de "história tradicional da psicologia" (YOUNG, 1966; DANZIGER, 1979, 1984, 1993; O'DONNELL, 1979; RICHARDS, 2002; ROGER, 2005; ROSE, 2008; TOOMELA, 2010).

A História Tradicional da Psicologia, que se tornou uma forma de "história oficial" da Psicologia, parte de um questionamento historiográfico que se ancora nas normas hodiernamente vigentes para definir o objeto de estudo da Psicologia e com ênfase em autores e idéias. O marco inaugural dessa corrente historiográfica é o livro História da Psicologia Experimental (BORING, 1950), escrito por Edwin Boring e publicado originalmente em 1929. O propósito de Boring era apresentar uma Psicologia Unificada a partir de uma suposta hegemonia da Psicologia Experimental (O'DONNELL, 1979). As principais características da História Tradicional da Psicologia são:

1) Ênfase na obra de "grandes" personalidades da Psicologia (Individualismo): Robert Watson é o primeiro historiador da Psicologia a apresentar uma História da Psicologia a partir da contribuição de alguns autores, identificando as principais contribuições destes pensadores ao campo psicológico e também estudando a biografia e o desenvolvimento intelectual destas figuras. O estudo biográfico pode servir para uma análise ampla de uma determinada época e de um contexto cultural específico. Infelizmente não é isso que ocorre com os estudos da História da Psicologia, que tradicionalmente se limita a investigar "grandes homens" e suas contribuições teóricas à Psicologia. Essa característica pode ser descrita como uma expressão do individualismo da cultura Ocidental 
na pesquisa em História da Psicologia ${ }^{1}$. Uma rara exceção a esse viés é o estudo de Leila Zenderland (1998), que analisa como os testes psicológicos se tornaram parte da cultura estadunidense a partir de um estudo sobre a vida e a obra de Henry Goddard.

2) Pouca importância atribuída ao contexto social (Internalismo): A concepção historiográfica tradicional tem produzido o que se denominou de "histórias internas" da Psicologia. O uso de ferramentas epistemológicas da Teoria do Conhecimento por parte de pesquisadores que eram predominantemente psicólogos e filósofos para analisar o desenvolvimento do conhecimento psicológico e os métodos de pesquisas desenvolvidos na Psicologia como se este envolver ocorresse na forma de um progresso gradual e linear até o presente, que se transforma em parâmetro para julgar o passado ${ }^{2}$. Outra característica importante do internalismo é apresentar a história como o desenvolvimento da racionalidade e com o triunfo do conhecimento científico frente à irracionalidade e a superstição.

3) História da Psicologia como História da Psicologia Estadunidense (Etnocentrismo): Em função da ascendência econômica e militar dos Estados Unidos, concomitantemente ao declínio da Cultura Alemã, o modelo de Psicologia que floresceu naquele país americano foi exportado para várias partes do planeta sob o rótulo de "A Psicologia". Os manuais de História da Psicologia, escritos por autores estadunidenses e traduzidos em vários países, expressam essa dominação: tomam como uma verdade inelutável que a História da Psicologia é a História da Psicologia nos Estados Unidos ${ }^{3}$ (PICKREN, 2009).

4) História da Psicologia como História da Psicologia Experimental, com pouca ou nenhuma preocupação com outras áreas da Psicologia e com a Psicologia Aplicada (Experimentalismo): Boring (1950) escreveu sua História da Psicologia Experimental para - entre outros objetivos - demonstrar que, embora durante algum tempo a Filosofia exercesse um papel importante para o desenvolvimento da Psicologia, havia chegado o momento que a presença do discurso filosófico na Ciência Psicológica se tornara anacrônica e obtusa (O'DONNELL, 1979) e propor, em um confronto com psicólogos que estavam desenvolvendo uma Psicologia Aplicada em várias áreas do campo social, uma Psicologia que produzisse um conhecimento essencialmente experimental derivado da pesquisa pura, sem nenhuma aplicação. Assim, seguindo o modelo de Boring, muitos manuais e pesquisas sobre História da Psicologia não apresentavam nenhuma informação sobre importantes áreas da Ciência Psicológica (Psicologia do desenvolvimento, Teoria da personalidade e Psicologia Social, por exemplo), o mesmo ocorrendo em relação a áreas aplicadas como Psicologia Clínica, Psicologia Organizacional, e Psicologia da Educação. 
A superação do monopólio dessa forma de produzir História produziu o que ficou conhecido como História Crítica da Psicologia. Danziger (1984) desenvolve uma análise de dois sentidos possíveis para a expressão "história crítica da psicologia". Inspirado no Programa Forte da Sociologia do Conhecimento ${ }^{4}$, que compreende 0 conhecimento científico como o resultado de um complexo processo de construção social de consensos entre especialistas acerca do que seja conhecimento legítimo, o psicólogo alemão distingue uma História Crítica da Psicologia em um sentido "Fraco" de uma em um sentido "Forte".

A História Crítica da Psicologia em um sentido "Fraco" se assenta, em três compromissos básicos: 1) um questionamento das autoridades e fontes primárias do campo; 2) analisar reflexivamente os pressupostos e compromissos do próprio historiador; 3) postura crítica em relação à Psicologia, questionando o caráter progressivo da produção do conhecimento psicológico.

Uma História Crítica da Psicologia em um sentido "Forte", contudo, pressupõe a construção de um marco historiográfico alternativo a partir de novas ferramentas conceituais: os processos construtivos dos objetos psicológicos (DANZIGER, 1984; 1993; 2003).

Danziger (1984) está interessado nas atividades construtivas (teóricas, práticas ou institucionais) que produzem objetos conceituais (o conceito de inconsciente, por exemplo), objetos técnicos (a psicoterapia breve, para citar um exemplo) e objetos sociais (o psicólogo hospitalar, para ilustrar com um exemplo tipicamente brasileiro).

Danziger (1979), fundamentado em Habermas (1990), propõe o conceito de interesses intelectuais, que define o ponto de contato entre os interesses sociais e as estruturas cognitivas. São os interesses intelectuais de uma comunidade científica que definem os conteúdos de sua disciplina. O conceito de interesse intelectual está relacionado ao de Profissionalização da Psicologia.

Há várias experiências de profissionalização que geraram modelos diferentes de Psicologia. Danziger (1979) compara os processos desenvolvidos nos Estados Unidos (Autonomia da Psicologia em relação à Filosofia) com o da Alemanha (projeto de um programa de investigação psicológica sem ruptura com outros campos disciplinares). O Brasil pode ser visto como exemplo de um terceiro modelo: desenvolvimento de um projeto de Psicologia aplicada à Educação e Saúde, com um precário suporte de um incipiente sistema universitário, sem possibilidade de construção de uma autonomia profissional até a década de cinquenta.

Outra categoria importante proposta por Danziger (1984) é de problemática. Diferentemente de problema, que remete a interesses individuais, esta categoria privilegia o estudo de sujeitos coletivos, buscando ir além das auto-representações que os psicólogos 
constroem de suas práticas e produções mediante as quais constroem seus objetos psicológicos.

A expressão "contexto social" é inadequada para designar essa compreensão da problemática, pois expressa uma concepção naturalista fundamentada na relação de um organismo com seu ambiente. Sujeitos coletivos formam um grupo social e/ou uma tradição intelectual, cujo trabalho reproduz uma tradição intelectual ou social em que estão imersas as práticas produtoras de objetos psicológicos.

Outra característica da História Crítica de Danziger é sua preocupação com o impacto no desenvolvimento teórico da disciplina produzido pela pesquisa histórica. História e Teoria Psicológicas estariam imbricadas, sendo o conhecimento histórico uma ferramenta importantíssima para desnaturalizar os objetos psicológicos.

O objetivo deste ensaio é analisar a produção historiográfica brasileira sobre a inserção da Psicologia no Contexto Hospitalar à luz dos conceitos de História Crítica de Danziger. O texto divide-se em quatro partes: Inicialmente, uma revisão sobre os estudos acerca da Psicologia da Saúde no contexto hospitalar é apresentada; o núcleo central do trabalho é a segunda seção, na qual se apresenta uma análise das pesquisas sobre a História da Psicologia no contexto hospitalar; na próxima seção se busca repensar a pesquisa histórica sobre a Psicologia no contexto hospitalar a partir de uma História Crítica da Psicologia da Saúde. Algumas considerações finais são apresentadas com o objetivo de recapitular e sintetizar as discussões feitas.

\section{A Psicologia da Saúde no contexto hospitalar brasileiro}

Os primeiros estudos sobre o trabalho do psicólogo no contexto hospitalar brasileiro apareceram no final da década de oitenta (LAMOSA, 1987; CAMPOS, 1988). Lamosa (1987) sugere que o psicólogo deve desenvolver uma imagem mais ampla como profissional de saúde, não se restringindo a questões de Saúde Mental. Campos (1988) propõe que o psicólogo, enquanto profissional de saúde, tenha um papel clínico, social, organizacional e educacional.

De certa forma, essa discussão se insere em um contexto mais amplo no qual a relação da Psicologia com o campo da Saúde é ressignificado e problematizado no Brasil. Alguns trabalhos publicados ainda na década de oitenta iniciaram a discussão sobre a atuação do psicólogo no contexto da saúde (MEJIAS, 1984; BRAGA CAMPOS, 1988; SILVA, 1988).

Na década de noventa, a discussão sobre a relação da Psicologia ganha maior divulgação a partir do lançamento do livro "Psicologia e 
Saúde: repensando práticas", publicado em 1992. A reflexão sobre a inserção da Psicologia no âmbito sanitário brasileiro ganhou uma maior visibilidade. Um dos textos mais influentes do livro foi o de Mary Jane Spink (1992), que versou sobre a estruturação do campo da Psicologia da Saúde. Embora não contenha nenhum texto específico sobre o trabalho do psicólogo no contexto hospitalar, "Psicologia e Saúde: repensando práticas", pode ser considerado o marco inaugural de uma Psicologia Crítica da Saúde no contexto brasileiro.

A produção de livros sobre o que se denominou de Psicologia Hospitalar, contudo, começou a ocorrer ainda na primeira metade da década de oitenta (ANGERAMI-CAMON, 1984). O psicólogo Valdemar Augusto Angerami, mais conhecido como Camon, publica uma série de livros sobre o tema durante a década de noventa (ANGERAMICAMON, 1995; 1996; 1997; 1998), sendo um dos autores mais influentes do assim denominado campo da Psicologia Hospitalar.

Em 1998, Yamamoto e Cunha publicam um artigo no qual, a partir de uma pesquisa empírica, apresentam alguns questionamentos ao campo. Os autores apontam para a necessidade de se repensar a formação do psicólogo brasileiro em função dos desafios do campo da saúde e criticam a constituição de uma Psicologia Hospitalar, defendendo que se situe o trabalho do psicólogo dentro da perspectiva da Psicologia da Saúde tomada em um sentido mais amplo. Em um artigo posterior, Yamamoto, Trindade e Oliveira (2002) reafirmam a inadequação do uso de um local de trabalho para designar uma área de atuação e acrescentam que tal prática tenderia a pulverizar e fragmentar o campo profissional da Psicologia, tornando assim muito difícil a construção de uma identidade profissional da Psicologia da Saúde.

O trabalho mais sistemático sobre o campo de atuação do psicólogo que trabalha em hospitais, no que tange ao entendimento da identidade profissional, foi o de Castro e Bornholdt (2004), que compreendem a denominada Psicologia Hospitalar como pertencente à área de Saúde, que utilizaria conhecimentos da Psicologia Clínica, das Ciências Biomédicas e da Psicologia Comunitária para intervir nos mais diversos contextos sanitários, incluindo o hospital. Outra contribuição importante do artigo é que ele é um dos primeiros a tratar da especificidade da Psicologia Hospitalar no Brasil tendo como parâmetro a realidade internacional. As autoras comparam nossa realidade com as realidades estadunidense e espanhola, nas quais 0 marco conceitual da Psicologia da Saúde serve de fundamentação teórica e prática para o trabalho no contexto hospitalar.

Uma crítica ao trabalho de Castro e Bornholdt (2004) é que as autoras apresentam a Psicologia da Saúde como se esta fosse uma área homogênea. De acordo com Crossley (2000) e Teixeira (2004), há pelo menos duas perspectivas teóricas atualmente: uma Psicologia 
da Saúde Tradicional e uma Psicologia da Saúde Crítica. Essa discussão, contudo, é praticamente inexistente no Brasil.

Seild e Costa (1999) publicaram um estudo sobre o trabalho do psicólogo na rede pública de Brasília no qual descreveram o modo como os psicólogos que atuavam em hospitais desenvolviam suas atividades. A pesquisa constatou a existência de dois modelos de atuação: o modelo clínico, caracterizado por atendimentos individuais, com pouca ou nenhuma interação com equipes de saúde. O modelo de atenção integral à saúde, em contraste, apresenta uma atuação difusa em diversos setores do hospital, em interação constante com os demais profissionais da saúde, visando atender pacientes e seus familiares, equipe e a comunidade em geral.

A pesquisa de Seild e Costa (1999) apontou também que os psicólogos que atuavam de acordo com o modelo de atenção integral eram caracterizados por realizarem mais pesquisas e pelo maior interesse em contribuir para a construção de um corpo teóricoprático da Psicologia da Saúde. Não há nenhum outro estudo a respeito da existência desses dois modelos, de modo que não é possível generalizar esse resultado e discutir os modelos de atuação existentes no Brasil. A crítica a uma abordagem clínica no contexto hospitalar, todavia, aparece em alguns trabalhos da área. (YAMAMOTO; CUNHA, 1998; MARCON; LUNA; LISBÔA, 2004; CASTRO; BORNHOLDT, 2004; SÁ, et al., 2005; ). Um aprofundamento dessa crítica, contudo, precisa considerar alguns dados apresentados em algumas pesquisas, que apontam a Psicanálise como a abordagem predominante e a Psicoterapia Breve como a técnica mais utilizada no contexto hospitalar. (YAMAMOTO; CUNHA, 1998; MARCON; LUNA; LISBÔA, 2004;). Os psicanalistas têm produzido uma reflexão sobre a prática da teoria criada por Freud no contexto hospitalar que já se avoluma (MOREIRA; PAMBLONA, 2006; COUTO, 2007; DUTRA; FERRARI, 2007; MOURA; SOUZA, 2007; PINHEIRO; VILHENA, 2007; GOMES, 2008; PISETTA, 2008). Infelizmente essa literatura não é utilizada nos estudos sobre a prática psicológica no hospital, o que possibilitaria uma discussão mais polifônica sobre a questão. Os estudos mais recentes sobre o campo hospitalar são muito específicos e não abordam a questão da fundamentação teórica da prática profissional (MORE, et al., 2009; SANTOS; JACÓ-VILELA, 2009).

Por fim, existe uma incipiente discussão a respeito da atuação do psicólogo em equipes multidisciplinares (FOSSI; GUARESCHI, 2004; TONETTO; GOMES, 2007). Vasconcelos (2002), contudo, prefere denominar de práticas pluriauxiliares aquelas em que ocorre a utilização de contribuições de um ou mais campos de saber para o domínio de um deles já existente, que se posiciona como coordenador dos demais. No âmbito do Conhecimento, o impacto dessa prática na área da Saúde ocorre na forma de um imperialismo 
epistemológico; no campo da prática, na forma de medicalização do social, sempre como decorrência da hegemonia da racionalidade médica (VASCONCELOS, 2002; TESSER, 2006a; 2006b; 2009; TESSER; LUZ, 2008).

Almeida (2000) questiona o imperialismo epistemológico no hospital geral, propondo uma interlocução entre os saberes que respeite a especificidade de cada um. A autora afirma que o trabalho do psicólogo no hospital geral não deve se limitar a resolução de conflitos, mas também deve ocorrer visando à promoção da saúde dos pacientes. Em suas palavras,

\begin{abstract}
Pensar a inserção do psicólogo no hospital geral, especialmente numa instituição pública, não pode dispensar a reflexão sobre a situação do sistema público de saúde, sua organização, as possibilidades de acesso da população aos serviços, as condições em que se dá o trabalho dos profissionais, as características sociais da população atendida, enfim, o conhecimento e a articulação de todos os fatores envolvidos no processo saúde-doença. (ALMEIDA, 2000, p. 27)
\end{abstract}

\title{
3 Análise das pesquisas sobre a História da Psicologia no contexto hospitalar
}

Três dissertações de Mestrado foram selecionadas para a análise de pesquisas sobre a História da Psicologia no Contexto hospitalar: A inserção do Psicólogo no Hospital Geral: a construção de uma nova especialidade, de Fernanda Martins Pereira, defendida em 2003; O Percurso histórico do serviço de Psicologia do hospital de Clínicas de Porto Alegre, escrito por Leda Pibernat Pereira da Silva e A construção de um lugar para a Psicologia em Hospitais de Sergipe, de Célia Maria Alcântara Machado Vieira. As duas últimas dissertações foram defendidas em 2006.

O trabalho de Pereira (2003) investigou o caminho percorrido desde o ingresso das primeiras psicólogas no Hospital dos Servidores do Estado do Rio de janeiro (HSE) em 1976 (setor de Pediatria) até a oficialização do Serviço de Psicologia do referido hospital ocorrido em 1983. Estruturado em três capítulos, a dissertação de Fernanda Pereira apresenta, inicialmente, um breve histórico da prática profissional dos psicólogos no Brasil; a autora se fundamenta na Sociologia das Profissões, principalmente no trabalho de Freidson (1996, 1998), para construir uma periodização dividida em três momentos, todos estruturados em torno do conceito de profissionalização: o primeiro, denominado de pré-profissional, é compreendido entre a criação das faculdades de medicina na Bahia e no Rio de Janeiro (1833) e o final do século XIX (1890), quando 
ocorre a Reforma Benjamin Constant da Educação ${ }^{5}$. De acordo com a autora

\begin{abstract}
A Psicologia não era uma prática definida ou regulamentada. O mercado de trabalho era incipiente. As associações profissionais e de pesquisa não foram identificadas. O que havia eram pessoas interessadas pelos temas e questões psicológicas. Não havia, portanto, a profissão de psicólogo no Brasil durante o século XIX. (PEREIRA, 2003, p. 15)
\end{abstract}

O segundo momento, denominado profissionalização, é compreendido entre 1890/1906 e 1975. Pereira afirma que Ele abrange desde a gênese da institucionalização da prática
psicológica até a regulamentação da profissão e a criação dos
seus dispositivos formais. Serão considerados como marcos
desse período: a Reforma Benjamim Constant (1890), a
inauguração dos laboratórios de psicologia experimental na
educação (1906) e a criação do código de ética (1975). A
partir de então, a psicologia passou a ter um conhecimento
próprio, institucionalizado e reconhecido, tornando-se
detentora de um determinado mercado de trabalho, ainda
que compartilhado com a medicina e a educação. (2003, p.
15)

O terceiro momento inicia-se com a criação do Código de Ética (1975) e encontra-se inconcluso. É denominado profissional e a partir de então a profissão passa por uma série de alterações com a proliferação de faculdades de Psicologia que lançam um número crescente de profissionais anualmente, contribuindo para a desvalorização da mão de obra. Novos espaços e práticas denominadas "emergentes" começaram a se constituir. Um desses novos espaços foi o hospital.

No segundo capítulo, Pereira apresenta algumas características da instituição hospitalar ao longo da História, com ênfase no estudo do Hospital dos servidores do Rio de Janeiro. Ela apresenta, inicialmente, as principais mudanças sofridas pela instituição hospitalar até que ela adquirisse sua configuração atual. Essa História Hospitalar se divide em quatro grandes momentos: mundo Greco-romano, I dade média, I dade moderna e I dade contemporânea. Em seguida, uma história do HSE é apresentada, descrevendo e analisando o seu surgimento no final da década de quarenta e o seu desenvolvimento até o momento de inserção das primeiras psicólogas no Serviço de Pediatria, no final dos anos setenta. A autora utilizou fontes primárias (documentos) e depoimentos orais de médicos que trabalharam naquela instituição hospitalar durante o período supramencionado. Cinco referenciais guiaram a análise da autora: 
O primeiro diz respeito às diferentes funções dos hospitais ao longo do tempo; o segundo ao lugar do médico nessas instituições; o terceiro, à forma de organização do trabalho; o quarto ao papel da tecnologia; e, finalmente, o quinto à clientela. A escolha desses referenciais apóia-se na crença de que estes são elementos fundamentais para a compreensão da estrutura e organização de qualquer instituição hospitalar. Além disso, são importantes para o entendimento das relações de poder existentes dentro dos hospitais, independente de sua época. O que muda é o significado de cada um desses elementos de acordo com o contexto histórico considerado. (PEREIRA, 2003, p. 34)

Pereira analisou a prática profissional das duas psicólogas pioneiras no HSE em seu terceiro capítulo. Ela procurou compreender 0 processo de inserção dessas profissionais no hospital, as atividades que elas desenvolveram e a existência de conflitos interprofissionais nas equipes de saúde e as estratégias utilizadas pelas entrevistadas para garantirem sua autoridade e autonomia profissionais.

Em sua conclusão, comentando as necessidades do psicólogo que atua no campo da saúde, a autora afirma:

Primeiramente, é necessário que conheça a história desse
campo de atuação. É preciso entender que o movimento da
psicologia hospitalar no Brasil, apesar de intenso, é um
processo recente e determinado pelo seu contexto. O
trabalho dos psicólogos nos hospitais do Rio de Janeiro com
certeza difere de outras regiões. Não é muito difícil
imaginarmos, inclusive, a inexistência dessa especialização
em alguns estados de nosso país. Além disso, o psicólogo
encontrará mais ou menos obstáculos dependendo do
hospital em que se encontra. Público ou privado, civil ou
militar, geral ou psiquiátrico: todas essas variáveis
interferem no trabalho hospitalar. (PEREIRA, 2003, p. 70)

A autora utilizou a Metodologia da História Oral como um método para realizar as entrevistas do segundo e do terceiro capítulos. A reflexão desenvolvida pela autora sobre o seu método de pesquisa limitou-se à comparação feita entre o documento oral e o escrito.

Silva (2006) pesquisou o percurso histórico do Serviço de Psicologia do Hospital das Clínicas de Porto Alegre (HCPA). Essa autora fez uma longa discussão sobre a História Oral como Método, que pode ser resumida nos seguintes pontos:

1) A História Oral permite que se obtenha dados que não estão registrados por algum tipo de documentação;

2) A Historia Oral investiga fatos contemporâneos, permitindo a convivência com as testemunhas vivas e a possibilidade de registro da memória dessas pessoas em sua própria voz;

3) A História Oral é um método que, além de produzir novos objetos de estudos e nova documentação, estabelece uma relação dialogal 
entre o historiador e os sujeitos da História, sendo também marcada pela intencionalidade do pesquisador na produção de documentos históricos.

Silva (2006) também apresenta uma avaliação das críticas feitas à História Oral como método. A defectibilidade da memória, a proximidade temporal com o objeto de estudo, a falta de precisão na forma e na cronologia e o uso, com certa freqüência, de apenas uma testemunha. Em relação à questão da memória, a autora, baseada em Massimi (2002), Batista (2003) e Olmos (2003), aponta que nas pesquisas em História da Psicologia no Brasil, em função da inexistência de documentos escritos, os estudiosos têm recorrido freqüentemente às narrativas dos atores que construíram essa História. Silva (2006) assim conclui sua reflexão sobre o uso da História Oral nas pesquisas historiográficas sobre a Psicologia no Brasil:

\begin{abstract}
A pesquisa baseada na memória e na oralidade é a chance de retomar o passado pela palavra, antes que ele desaparece no silêncio e no esquecimento, além de permitir a composição de uma narrativa coletiva e pública da experiência de psicólogos no Brasil, mesmo que essa não represente uma verdade definitiva e absoluta, mas algo relativo à versão dos narradores (p. 19).
\end{abstract}

A dissertação de Silva (2006) divide-se em três partes: na primeira, a autora apresenta o método da História Oral, descrevendo suas características, particularidades e limitações; na segunda, a História do Serviço de Psicologia do Hospital das Clínicas de Porto Alegre é apresentada, antecedida por uma revisão sobre as origens da prática psicológica em Hospitais nos Estados Unidos e no Brasil. Na terceira e última parte, Silva (2006) apresenta as discussões e considerações finais sobre sua pesquisa. Como a questão do método tratada na primeira parte já foi comentada neste artigo, vamos nos deter às duas últimas partes da dissertação de Silva (2006).

A segunda parte da dissertação de Silva (2006), por sua vez, apresenta uma dupla face da História da Psicologia no HCPA: uma referente ao trabalho em Psicologia Organizacional e a outra referente ao trabalho em Psicologia Clínica.

O desenvolvimento de uma Psicologia Organizacional no HCPA não tem paralelo nos contextos hospitalares investigados nos outros estudos históricos que são discutidos neste artigo, assim como nas pesquisas sobre a atuação dos psicólogos nos hospitais que foram citados na seção anterior deste texto. Essa é uma das principais contribuições da autora: mostrar que a História da Psicologia no contexto hospitalar não se confunde com história da extensão da Psicologia Clínica àquele contexto. 
O trabalho de Vieira (2006) difere dos dois primeiros em vários aspectos. A principal diferença é uma pesquisa histórica que se volta para uma Região Geográfica (no caso Sergipe) como objeto de estudo, enquanto as pesquisas de Pereira (2003) e Silva (2006) apresentam uma investigação sobre a História da Psicologia Hospitalar em um contexto institucional específico. Outra peculiaridade da dissertação de Vieira (2006) é o deslocamento que faz em relação ao Sudeste, região onde surgiu a psicologia Hospitalar, voltando-se para a Região Nordeste, que segundo a autora é pouco estudada pelos estudiosos da História da Psicologia no Brasil $^{6}$. Em suas palavras,

\begin{abstract}
Pretendo dar visibilidade ao trabalho desenvolvido no menor Estado do país, apesar de não ter nenhuma pretensão de esgotar as possibilidades, mas de apresentar uma pequena parte daquilo que já está sendo feito, de modo particular a atuação dos psicólogos em hospitais, e, quem sabe, instigar os pesquisadores do nordeste e de outras regiões do país a difundirem seu trabalho, contribuindo, dessa forma, para uma História da Psicologia que realmente seja representativa do Brasil, e não regionalizada. (p. 51)
\end{abstract}

A dissertação de Vieira (2006) apresenta quatro capítulos: no primeiro, a autora traça um percurso histórico das instituições de cuidado aos doentes, percorrendo a linha do tempo desde os templos antigos até a constituição do hospital contemporâneo. No segundo, discute a História da Psicologia no Brasil, tendo como foco a relação entre Psicologia e medicina, partindo da época colonial até o momento atual. A autora trata também da história da inserção dos psicólogos em Hospitais no Brasil. No terceiro capítulo é apresentada a inserção dos psicólogos no contexto hospitalar em Sergipe, a partir de uma pesquisa empírica na qual foram entrevistados 28 psicólogos. No quarto capítulo, uma cronologia da inserção dos psicólogos nos hospitais em Sergipe é apresentada, seguida de uma discussão sobre o perfil da atuação do psicólogo no contexto hospitalar sergipano comparativamente com resultados de pesquisas em outros estados, assim como traz também uma reflexão sobre perspectivas, possibilidades e desafios para o futuro deste trabalho do psicólogo em Sergipe. A autora se utilizou metodologicamente de fontes secundárias (os estudos historiográficos) e de fonte primária, a memória dos psicólogos entrevistados.

Duas outras características desta dissertação também merecem ser mencionadas: a compreensão de um sujeito coletivo como objeto de estudo e uma análise da situação atual da psicologia hospitalar em Sergipe, o que faz desta pesquisa uma História do Presente? 


\section{Repensando a pesquisa histórica sobre a psicologia no contexto hospitalar}

Uma avaliação sinóptica desta literatura estudada indica, antes de tudo, que o tema carece de maior quantidade de trabalhos. É imprescindível que se tenha mais pesquisas sobre a História da Psicologia Hospitalar no Brasil. Uma análise das dissertações encontradas nos permite afirmar que há dois modelos de História da Psicologia Hospitalar: uma história institucional (o de Fernanda Pereira sobre o hospital dos servidores do Rio de Janeiro e o de Leda Silva sobre o Hospital de Clínicas de Porto Alegre) e uma história territorial (o de Célia Vieira sobre os hospitais em Sergipe). Os dois modelos são importantes, mas parece que uma história institucional da psicologia hospitalar seria mais bem adequada para os grandes centros, enquanto que uma história territorial da psicologia hospitalar seria mais bem indicada para os centros menores, principalmente no interior ou em alguns estados do Norte-Nordeste. Algumas questões precisam ser avaliadas:

1) O uso da Sociologia das profissões em pesquisas de História da Psicologia. O trabalho de Pereira (2003) foi o primeiro a utilizar a Sociologia das profissões no estudo da Historia da Psicologia Hospitalar no Brasil. Esse é seu grande mérito. Mas alguns problemas são apresentados pela análise que a autora realizou. Em primeiro lugar, sua proposta de periodização da História da Psicologia apresentada no primeiro capítulo da dissertação e em um artigo (PEREIRA; PEREIRA NETO, 2003) - é inadequada, pois constrói um modelo de periodização a partir da profissionalização da Psicologia, desconsiderando as especificidades de cada período. Assim, a autora divide a história da Psicologia no Brasil em três períodos: préprofissional (1834-1890), profissionalização (1890/1906-1975) e profissional $(1975)$. Essa proposta pretende refinar o modelo de Pessotti (1988), que divide a História da psicologia no Brasil em quatro períodos, a saber: pré-institucional (1500-1833), institucional (1833-1934), universitário (1934-1962) e profissional (1962). Os dois modelos não conseguem explicitar a tensão existente entre duas histórias da Psicologia no Brasil: a História do Conhecimento Psicológico e a História da Psicologia como profissão. Danziger pode servir de referência aqui. A História da Psicologia como Conhecimento estuda as idéias psicológicas, as teorias psicológicas, os objetos de estudo da Psicologia e a linguagem Psicológica. A História da Psicologia como Profissão estuda os modelos profissionais, os papéis sociais gerados pelas práticas dos psicólogos, a regulamentação estatal da prática profissional e os embates com outras profissões. A História da Psicologia como profissão se inicia na década de trinta com Radecki (CENTOFANTI, 1982). Bernardes (2004) resgata alguns textos que discutem a formação do psicólogo e mostra como o 
período de profissionalização da psicologia ocorre entre 1932 até o final da década de 50. Assim, a psicologia no contexto hospitalar surge, efetivamente, em um período em que o processo de profissionalização da psicologia já estava bastante avançado. Uma compreensão da dinâmica que fez surgir a psicologia no contexto hospitalar, contudo, somente poderá ser compreendida se levarmos em conta as especificidades desta instituição. A sociologia das profissões pode ser útil aqui se trabalharmos a partir de contextos institucionais específicos, como na proposta de Graça Carapinheiro de uma "sociologia dos serviços hospitalares" (CARAPINHEIRO, 1991; 1993; 2008). Lopes (2006) oferece um modelo teórico de análise sociológica que pode ser utilizado em pesquisas históricas no contexto hospitalar, enfocando o que ela denomina de "trilogia analítica": o trabalho em equipe, a autonomia profissional e o isolamento no trabalho. Embora algumas pesquisas tenham sido produzidas sobre 0 trabalho do psicólogo em equipes multidisciplinares no contexto hospitalar (FOSSI; GUARESCHI, 2004; TONETTO; GOMES, 2007), os estudos não utilizaram o referencial teórico da sociologia das profissões.

2) A Psicologia Organizacional no contexto hospitalar. O trabalho de Silva (2006) nos traz uma rara discussão sobre o trabalho do psicólogo organizacional no contexto hospitalar, um dos méritos dessa dissertação. Silva (2006) não teoriza sobre a questão do poder que é pano de fundo para a primeira extinção do serviço de psicologia, como também não se aprofunda sobre as mudanças na forma de trabalho do psicólogo (ênfase em avaliação psicológica sendo substituída por uma preocupação com a saúde do trabalhador e pelas relações de trabalho). Esta mudança foi provocada por uma decisão judicial que aboliu a avaliação psicológica em concursos públicos no HCPA em 1993. Embora tenhamos vários estudos sobre o desenvolvimento da Psicologia Organizacional em nosso país (ZANELLI; BASTOS, 2004), não há estudos significativos sobre a psicologia organizacional no contexto hospitalar. Assim como também os estudos sobre Psicologia no contexto hospitalar não problematizam o trabalho do psicólogo organizacional no hospital.

3) A institucionalização da psicologia no contexto hospitalar. Um dos critérios históricos utilizados para definir uma subdisciplina é a publicação de um manual ou livro-texto que apresente as noções básicas, métodos e precursores históricos do novo campo de pesquisa e/ou prática (GOUREVITCH, 1995; LOMBARDO; FOSCHI, 2003). O desenvolvimento da Psicologia no contexto hospitalar ocorreu a partir da divulgação de uma literatura essencialmente nacional, o que justifica a produção de uma "história do livro de psicologia hospitalar no Brasil". Além disso, é necessário que se produza pesquisas sobre as sociedades científicas de Psicologia no contexto hospitalar e dos periódicos especializados dedicados a este campo. 


\section{Considerações Finais}

Embora as pesquisas sobre a História da Psicologia no Brasil tenham aumentado nos últimos anos, não há muitas pesquisas sobre o desenvolvimento das áreas mais consolidadas de aplicação da psicologia. Compreender a forma de "profissionalização" da Psicologia Brasileira exige que se repense a centralidade do mundo acadêmico como espaço de desenvolvimento da psicologia. Na terminologia desenvolvida por Danziger (1984), é necessário que investiguemos mais as atividades construtivas que produzem objetos técnicos e sociais do que as que produzem objetos conceituais. A escassa produção sobre a história da psicologia no contexto hospitalar tem o mérito de tornar essa um objeto de estudo histórico. Além disso, questões como a profissionalização da psicologia, a sociologia das profissões, a presença da psicologia organizacional no contexto hospitalar, a questão da diversidade regional brasileira, entre outras, são contribuições importantes para a compreensão dos objetos sociais produzidos pela prática psicológica (a questão da psicologia como profissão de saúde no contexto hospitalar). A História Institucional e a História Territorial, duas possíveis formas de produção historiográfica sobre a psicologia no contexto hospitalar, podem ajudar a desenvolver tanto o campo da Psicologia da Saúde, quanto a própria historiografia da psicologia no Brasil. As idéias de Danziger sobre profissionalização, sujeitos coletivos e atividades construtivas de objetos certamente serão de grande valia no desenvolvimento de mais pesquisas.

\section{Referências}

ALMEIDA, E. O psicólogo no hospital geral. Psicologia: Ciência \& Profissão, Brasília, v. 20, n. 3, p. 24-27, 2000.

ANGERAMI-CAMON, V. (Org.). Psicologia Hospitalar: a atuação do psicólogo no contexto hospitalar. São Paulo: Traço, 1984.

Pioneira, 1995.

(Org.). Psicologia Hospitalar: teoria e prática. São Paulo:

Pioneira, 1997.

(Org.). E a Psicologia entrou no hospital. São Paulo:

(Org.). Urgências psicológicas no hospital. São Paulo: Pioneira, 1998.

ANGERAMI-CAMON, V.; CHIATTONE, H.; NICOLETTI, E. (Orgs.). 0 doente, a Psicologia e o hospital. São Paulo: Pioneira, 1996. 
AREOSA, J.; CARAPINHEIRO, G. Quando a imagem é profissão: profissões da imagiologia em contexto hospitalar. Sociologia, Problemas e Práticas, Lisboa, v. 12, n. 57, p. 57-108, 2008.

ASH, M.; WOODWARD, R. (Orgs.). Psychology in twentiethcentury: thought and society. Cambridge: Cambridge University Press, 1989.

BATISTA, M. As relações entre identidade, memória e pesquisa da história da Psicologia. Memorandum, Belo Horizonte, n.4, p. 33-39, 2003.

BERNARDES, J. O debate atual sobre a formação em Psicologia no Brasil: permanências, rupturas e cooptações políticas. 2004. 207f. Tese (Doutorado em Psicologia Social) - Programa de Pós-Graduação em Psicologia Social, Pontifícia Universidade Católica, São Paulo, 2004.

BLOOR, D. Knowledge and social imagery. Chicago: University Press, 1991.

Ideals and monisms: recent criticisms of the Strong Programme in the Sociology of knowledge, Studies in History and Philosophy of Science, v. 38, n. 1, p. 210-234, 2007.

BORING, E. A history of Experimental Psychology. 2.ed. New York: Appleton, 1950.

BRAGA CAMPOS, F. O psicólogo, o usuário e a saúde mental na instituição pública: o atendimento na rede básica de saúde de Campinas. 1988. Dissertação (Mestrado em Psicologia) - PósGraduação em Psicologia Social, Pontifícia Universidade Católica, São Paulo, 1988.

BROCK, A. (Org.). I nternationalizing the History of Psychology. New York: New York University Press, 2006.

CAMPOS, T. 0 psicólogo em hospitais: aspectos de sua atuação em hospital geral. 1988. (Doutorado em Psicologia) - PósGraduação em Psicologia Social, Pontifícia Universidade Católica, São Paulo, 1988.

CAPSHEW, J. Psychology on the march: science, practice, and professional identity in America, 1929-1969. Cambridge: Cambridge University Press, 1999.

CARAPINHEI RO, G. Poder médico e poder administrativo no cotidiano hospitalar. Revista Crítica de Ciências Sociais, Lisboa, n.33, p. 8391, 1991.

Saberes e poderes no hospital: uma Sociologia dos serviços hospitalares. Porto: Afrontamento, 1993.

CARVALHO, D. A cidade e a alma reinventadas: a história da Psicologia como signo e vetor da modernização urbana. São Paulo: Casa do Psicólogo, 2010.

CASTRO, E.; BORNHOLDT, E. Psicologia da saúde $x$ Psicologia hospitalar: definições e possibilidades de inserção profissional. 
Psicologia: Ciência \& Profissão, Brasília, v. 24, n. 3, p. 48-57, 2004.

CENTOFANTI, R. Radecki e a Psicologia no Brasil, Psicologia: Ciência e profissão, Brasília, v. 3, n. 1, p.2-50, 1982.

COUTO, L. O analista Sinthoma: uma das funções do analista no hospital geral. Epistemo-Somática, Belo Horizonte, v. 4, n. 1, p. 93-109, 2007.

CROSSLEY, M. Rethinking health Psychology. Buckingham: Open University Press, 2000.

CUSHMAN, P. Constructing the self, constructing America: a cultural History of Psychotherapy. Reading: Addison Wesley, 1995.

DANZIGER, K. The social origins of modern Psychology. In: BUSS, A. (Org.). Psychology in Social Context. New York: Irvington, 1979. p. 25-44.

Towards a conceptual framework for a critical History of Psychology. Historia de la Psicología, Valencia, v. 5, n. 1-2, p. 99107, 1984.

Three challenges for the History of Psychology. Invited address presented to Division 26 of the American Psychological Association, (Mimeo), 1993.

Constructing the subject: historical origins of Psychological research. Cambridge: Cambridge University Press, 1994.

DERKSEN, M. Discipline, subjectivity and personality: an analysis of the manuals of four psychological tests. History of the Human Sciences, v. 14, n. 1, p. 25-47, 2001.

DUMONT, L. O Individualismo: uma perspectiva antropológica da ideologia moderna. Rio de Janeiro: Rocco, 1993.

DUTRA, A.; FERRARI, I. Um estudo sobre a Psicanálise aplicada em um hospital geral, Arquivos Brasileiros de Psicologia, Rio de janeiro, v. 59, n. 2, p. 270-282, 2007.

FERREIRA, M. História do tempo presente: desafios. Cultura Vozes, Petrópolis, v. 94, n. 3, p. 11-124, 2000.

FOSSI, L.; GUARESCHI, N. A Psicologia hospitalar e as equipes multidisciplinares. Revista da Sociedade Brasileira de Psicologia Hospitalar, Belo Horizonte, v. 7, n. 1, p. 29-43, 2004.

FREIDSON, E. Para uma análise comparada das profissões: a institucionalização do discurso e do conhecimento formais. Revista Brasileira de Ciências Sociais, São Paulo, v. 11, n. 31, p. 141-154, 1996.

$\overline{1} \overline{9} \overline{8}$.

Renascimento do profissionalismo. São Paulo: Edusp,

GAMBOA, A. Historia del presente: estado de la cuestión y conceptualización. Historia Actual Online, Cádiz, n. 3, p. 101-116, 2004. 
GEUTER, U. The professionalization of Psychology in Nazi Germany. Cambridge: Cambridge University Press, 1992.

GIBBY, R.; ZICKAR, M. A history of the early days of personality testing in American Industry: an obsession with adjustment. History of Psychology, v. 11, n. 3, p. 164-184, 2008.

GOMES, D. O corpo psicanalítico no hospital. 2008. $127 f$. Dissertação (Mestrado em Psicologia) - Programa de Pós-Graduação em Psicologia, Universidade Federal de Uberlândia, Uberlândia, 2008. GOUREVITH, M. Naissance d'une especialité medicale. In : GOUREVITCH, D. (Org.). Histoire de la medicine - leçons methodologique. Paris: Ellipses, 1995, p. 124-129.

GUTHRIE, R. Even the rat was white: a historical view of Psychology. Boston: Ally \& Bacon, 1998.

HABERMAS, J. Conocimiento e Interé. Madrid: Taurus, 1990.

HILGARD, E.; LEARY, D.; MCGUIRE, G. A História da Psicologia. In: BROZEK, J.; MASSIMI, M. (Orgs.). Historiografia da Psicologia Moderna - Versão Brasileira, São Paulo: Loyola, 1998. p.399-432. LAKATOS, I. La metodología de los programas de investigación científica. Madrid: Alianza, 1989.

História da ciência e suas reconstruções racionais. Lisboa: Edições 70, 1998.

LAMOSA, B. O psicólogo clínico no hospital: contribuição para o desenvolvimento da profissão no Brasil. 1987. Tese (Doutorado em Psicologia) - Programa de Pós-Graduação em Psicologia Social, Pontifícia Universidade Católica, São Paulo, 1987.

LOMBARDO, G.; FOSCHI, R. The concept of personality in $19^{\text {th }}$ century French and $20^{\text {th }}$ - century American Psychology. History of Psychology, v. 6, n. 2, p. 123-142, 2003.

MARCON, C.; LUNA, I.; LISBOA, M. O psicólogo nas instituições hospitalares: características e desafios. Psicologia: Ciência \& Profissão, Brasília, v. 24, n. 1, p. 28-35, 2004.

MASSIMI, M. Memória e História na História da Psicologia: dois exemplos de produção de documentos. Memorandum, Belo Horizonte, n. 2, p. 2-12, 2002.

MEJIAS, N. O psicólogo, a saúde pública e o esforço preventivo. Revista de Saúde Pública. São Paulo, v. 18, , p.155-161, 1984. MIGUEL, G. Historia y presente. Berceo, n. 140, p. 293-326, 2001. MORE, C.; CREPALDI,M.; GONÇALVES, J.; MENEZES, M. Contribuições do pensamento sistêmico à prática do psicólogo no contexto hospitalar. Psicologia em Estudo, Maringá, v. 14, n. 3, p. 465-473, 2009.

MOREIRA, A.; PAMPLONA, C. Dispositivos clínicos em Hospital Geral. Psicologia Clínica, Rio de Janeiro, v. 18, n. 2, p. 13-24, 2006.

MOURA, M.; SOUZA, M. Psicanálise e hospital: se ao "a" deve o analista chegar, por onde andava ele? Epistemo-Somática, Belo Horizonte, v. 4, n. 2, p. 127-138, 2007. 
O'DONNELL, J. The crisis of experimentalism in the 1920s: E.G. Boring and his uses of History. American Psychologist, v. 34, n. 4, p. 289-295, 1979.

O'DONNELL, J. The origins of behaviorism: American Psychology, 1870-1920. New York: New York University Press, 1985.

OLMOS, J. A relação entre narração e memória como possibilidade metodológica na constituição da História da Psicologia no Brasil. Memorandum, Belo Horizonte, n. 4, p. 40-47, 2003.

PALMA-FILHO, J. A república e a educação no Brasil (1889-1930). In: PALMA FILHO, J. Pedagogia cidadã: cadernos de formação História da Educação. São Paulo: UNESP/Santa Clara Editora, 2005. p.49-60.

PEREIRA, F. A inserção do psicólogo no hospital geral: a construção de uma nova especialidade. Rio de Janeiro: FIOCRUZ, 2003.

PESSOTTI, I. Notas para uma História da Psicologia Brasileira. In: Conselho Federal de Psicologia (Org.). Quem é o psicólogo brasileiro? São Paulo: EDICON, 1988. p. 17-31.

PICKREN. W. Indigenization and the History of Psychology. Psychological Studies, n. 54, p. 87-95, 2009.

PINHEIRO, N.; VILHENA, J. Entre o público e o privado: a clínica psicanalítica no ambulatório hospitalar. Arquivos Brasileiros de Psicologia, Rio de Janeiro, v. 59, n. 2, p. 201-216, 2007.

PISETTA, M. Angústia e demanda de análise: reflexões sobre a Psicanálise no Hospital. Boletim de Psicologia, São Paulo, v. 58, n. 129, p. 171-183, 2008.

RICHARDS, G. 'Race', racism and Psychology: toward a reflexive History. London: Routledge, 1997.

. The Psychology of Psychology: A historically grounded sketch. Theory and Psychology, v. 12, n. 1, p. 7-36, 2002.

ROSA, P. Memória da Psicologia em Pernambuco, Recife: EdUFPE, 2001.

ROSE, N. Psicologia como uma ciência social. Psicologia \& Sociedade, Florianópolis, v. 20, n. 2, p. 155-164, 2008.

SÁ, A.; LIMA, A.; SANTOS, I,; CLEMENTE, L. Psicólogo hospitalar da cidade de Recife-PE: formação e atuação. Psicologia: Ciência \& Profissão, Brasília, v. 25, n. 3, p. 384-397, 2005.

SANTOS, F.; J ACÓ-VILELA, A. O psicólogo no hospital geral; estilos e coletivos de pensamento. Paidéia, Ribeirão Preto, v. 19, n. 43, p. 189-197, 2009.

SEID, E.; COSTA JR., A. O psicólogo na rede pública de saúde do Distrito Federal. Psicologia: Teoria e Pesquisa, Brasília, v. 15, n. 1, p. 27-32, 1999.

SILVA, L. O percurso histórico do serviço de psicologia do Hospital das Clínicas de Porto Alegre. 2006. 87f. Dissertação (Mestrado em Psicologia) - Programa de Pós-Graduação em 
Psicologia, Universidade Federal do Rio Grande do Sul, Porto Alegre, 2006.

SILVA, R. O trabalho do psicólogo em centros de saúde: algumas reflexões sobre as funções da Psicologia na atenção primária à Saúde. 1988. Tese (Doutorado em Psicologia) Programa de Pós-Graduação em Psicologia, Universidade de São Paulo, São Paulo, 1988.

SMITH, R. The History of psychological categories. Studies in History and Philosophy of Biological and Biomedical Sciences, Bloomington, v. 36, n. 1, p. 55-94, 2005.

SOKAL, M. (Org.). Psychological testing and American society: 1890-1930. New Brunswick: Rutgers University Press, 1987.

SPINK, J. Psicologia da saúde: a estruturação de um novo campo de saber. In: CAMPOS, F. (Org.). Psicologia e saúde: repensando práticas. São Paulo: Hucitec, 1992. p. 11-23.

STOLCKE, V. Gloria o maldición del individualismo moderno ségun Louis Dumont. Revista de Antropologia, São Paulo, v. 44, n. 2, p. 7-37, 2001.

TEIXEIRA, J. Psicologia da Saúde. Análise Psicológica, Lisboa, v. 22, n. 3, p. 441-448, 2004.

TESSER, C. Medicalização social (I): o excessivo sucesso do epistemicídio moderno na saúde. Interface, Botucatu, v. 10, n. 19, p. 61-76. 2006a.

- Medicalização social (II): limites biomédicos e propostas para a clínica na atenção básica. I nterface, Botucatu, v. 10, n. 20, p. 347-362, 2006b.

. Três considerações sobre a "má medicina". Interface, Botucatu, v. 13, n. 31, p. 273-286, 2009.

TESSER, C.; LUZ, M. Racionalidades médicas e integralidade. Ciência \& Saúde Coletiva, Rio de Janeiro, v. 13, n. 1, p. 195-206, 2008.

TONETTO, A.; GOMES, W. A prática do psicólogo hospitalar em equipe multidisciplinar. Estudos de Psicologia (Campinas), Campinas, v. 24, n. 1, p. 89-98, 2007.

TOOMELA, A. Culture of science: strange history of the methodological thinking in Psychology. Integrative Psychological and Behavioral Science, n. 41, p. 6-20, 2007a.

. History of methodology in Psychology: starting point, not the goal. Integrative Psychological and Behavioral Science, n. 41, p. 75-82, 2007b.

. Poverty of modern mainstream Psychology in autobiography: reflections on a History of Psychology in autobiography. Culture \& Psychology, v. 16, n. 1, p. 127-144, 2010.

VASCONCELOS, E. Complexidade e pesquisa interdisciplinar. Epistemologia e metodologia operativa. Petrópolis: Vozes, 2002.

VIEIRA, C. A construção de um lugar para a Psicologia em hospitais de Sergipe. 2006. 164f. Dissertação (Mestrado em 
Psicologia) - Pós-Graduação em Psicologia Social, Pontifícia Universidade Católica, São Paulo, 2006.

YAMAMOTO, O.; CUNHA, I. O psicólogo em hospitais de Natal: uma caracterização preliminar. Psicologia: Reflexão e Crítica, Porto Alegre, v. 11, n. 2, p. 345-362, 1998.

YAMAMOTO, O.; TRINDADE, L.; OLIVEIRA, I. O psicólogo em hospitais no Rio Grande do Norte. Psicologia USP, São Paulo, v. 13, n. 1, p. 217-246, 2002.

YOUNG, R. Scholarship and the History of the Behavioural Sciences. History of Science, n. 2, p. 1-51, 1966.

ZANELLI, J.; BASTOS, A. Inserção profissional do psicólogo em organizações e no trabalho. In: ZANELLI, J. C.; BORGES-ANDRADE, J. E.; BASTOS, A. V. B. (Orgs.). Psicologia, organizações e trabalho no Brasil. Porto Alegre: ArtMed, 2004, p. 466-491.

ZENDERLAND, L. Measuring minds: Henry Herbert Goddard and the origins of American Intelligence Testing. Cambridge: Cambridge University Press, 1998.

\section{Endereços para correpondência: \\ Denis Barros de Carvalho}

Rua Trevos, 1125, Morada do Sol, CEP 64056-210, Teresina-PI, Brasil.

Endereço eletrônico: denispsi@bol.com.br

Leylanne Martins Ribeiro de Souza

Rua Trevos, 1125, Morada do Sol, CEP 64056-210, Teresina-PI, Brasil.

Endereço eletrônico: superleylanne@hotmail.com

Lourena Silva Rosa

Rua Deputado J oão Martins do Rêgo, 5434, Santa Isabel, CEP: 64055-190, Teresina

- PI, Brasil.

Endereço eletrônico: psicologartista@hotmail.com

Máyra Laís de Carvalho Gomes

Rua Trevos, 1125, Morada do Sol, CEP 64056-210, Teresina-PI, Brasil.

Endereço eletrônico: mayra.lais@hotmail.com

Recebido em: 12/05/2011

Reformulado em: 27/07/2011

Aceito para publicação em: 28/07/2011

Acompanhamento do processo editorial: Ana Maria Jacó-Vilela

\section{Notas}

* Professor Adjunto do Departamento de Fundamentos da Educação da Universidade Federal do Piauí- UFPI, Doutor em Psicologia Social pela Universidade Federal do Rio Grande do Norte-UFRN.

**Acadêmicas de Psicologia da Universidade Estadual do Piauí-UESPI.

${ }^{1}$ Para uma discussão sobre o individualismo contemporâneo, ver Dumont (1993) e Stolcke (2001).

${ }^{2}$ Imre Lakatos $(1989,1998)$ é conhecido defensor da História Interna da Ciência. 
${ }^{3}$ Para uma discussão sobre a pluralidade de Psicologia em função da diversidade cultural, ver Brock (2006) e Toomela (2007a, 2007b, 2010).

${ }^{4}$ Para uma apresentação do "Programa Forte" da Sociologia do Conhecimento por um dos seus principais expoentes, ver Bloor $(1991,2007)$.

${ }^{5}$ Para mais informações sobre a Reforma Benjamin Constant, ver Palma Filho (2005).

${ }^{6}$ Há poucos livros sobre a História da Psicologia na Região Nordeste. Rosas (2001) e Carvalho (2010) são exemplos de uma literatura ainda incipiente.

${ }^{7}$ Sobre História do presente, ver Ferreira (2000), Miguel (2001) e Gamboa (2004). 